\section{Severe Life-Threatening Odontogenic Infections: Two Case Reports}

Kosba A $^{*}$, van Mierlo A, Dik E, Böckmann R and Kessler P

Department of Cranio-Maxillofacial Surgery, Maastricht University Medical Center, The Netherlands

\begin{abstract}
Despite greatly improved dental health care in more developed countries, severe life-threatening odontogenic infections still occasionally occur leading to hospitalization. A comprehensive quick diagnosis and immediate treatment are life-saving procedures. Here we report on two cases with severe septic complications due to odontogenic infections extending to the neck and mediastinum with airway obstruction that required immediate tracheostomy and drainage of all involved spaces. Advanced stages of odontogenic head and neck infections have to be addressed surgically immediately to drain abscess cavities properly. Special importance must be given to the airway maintenance. The antibiotic shielding serves to prevent the spreading of the infection, but is only supporting the surgical therapy.

Keywords: Abscess; Developed countries; Mediastinitis; Oral hygiene; Surgical decompression
\end{abstract}

\section{Introduction}

Severe odontogenic infections as a serious disease and cause of death have been known since antiquity [1]. It has been documented that most infections of the head and neck are of odontogenic origin [2]. The earliest stage of an odontogenic infection is the inoculation of bacteria into periapical or pericoronal tissues. The portal by which bacteria enter these tissues may be a carious or non-vital tooth, defective root canal treatment, or deep periodontal pocket. The periapical tissues are the initial site of bacterial growth, resulting in a focus of infection, which induces an immune response in the patient [3]. Occasionally, the immune system alone or together with a therapeutic intervention (e.g., dental treatment, antibiotics) suffices in combating the infection. At other times, however, the infection cannot be restrained and spreads along the path of the least resistance to affect the deep maxillofacial spaces formed by the architecture of the connective tissue layers of the perioral and neck regions.

*Corresponding author: Kosba A, Department of Cranio-Maxillofacial Surgery, Maastricht University Medical Center, P Debyelaan 25, 6202AZ Maastricht, The Netherlands, Tel: +31 623002864; E-mail: a_kosba@yahoo.com

Citation: Kosba A, van Mierlo A, Dik E, Böckmann R, Kessler P (2017) Severe Life-Threatening Odontogenic Infections: Two Case Reports. J Infect Non Infect Dis 3: 021.

Received: February 14, 2017; Accepted: March 22, 2017; Published: April 06 2017
Surgical incision to open and drain affected spaces is the cornerstone of therapy and it should only be postponed when vital signs warrant a safer operative course. Various general and local predisposing factors are known to increase the risk of odontogenic infection becoming severe. Such factors include unstable diabetes mellitus, immunosuppression and post radiotherapy [4]. There is no evidence that antibiotics are of benefit in otherwise healthy individuals $[5,6]$.

Severe odontogenic infections can be unpredictable in terms of their course. On admission, signs and symptoms vary from a simple local swelling to a life-threatening condition. Dramatic life-threatening conditions, such as Ludwig's angina, cervical cellulitis, mediastinitis and systemic complications, such as septicemia and cavernous sinus thrombosis, have been reported. Although such complications are rare in developed countries, they cannot be overlooked. Careful anamnesis, physical examination and laboratory tests are needed [7].

This article reports two late-presenting cases of deep neck infection, one of whom developed severe thoracic complications.

\section{Report of Cases}

\section{Case 1}

A 31-year-old gentleman reported to the Department of Cranio-Maxillofacial Surgery of the Maastricht University Medical Center (MUMC) with a chief complaint of pain and swelling in relation to the left lower jaw and neck since three days which was later accompanied with dysphagia. On physical examination, he had respiratory distress and was toxic in appearance, his vital signs were monitored immediately: temperature $38.0^{\circ} \mathrm{C}$; pulse rate 93 beats/minute, blood pressure $151 / 83 \mathrm{mmHg}$ and a respiratory rate of 25 breaths/minute. Mouth opening was limited to $1.5 \mathrm{~cm}$ and revealed a grossly decayed lower left second molar tooth. Extra-oral swelling was bilateral, indurated, nonfluctuant with involvement of the submandibular and sublingual regions. A Computed Tomography (CT) of the neck was done on the same day which revealed bilateral multiple collections in the neck more evident on the left side (Figure 1). The extension of the affected spaces reached the level of the aorta. The blood values were normal except for an elevated C-Reactive Protein (CRP) $446 \mathrm{mg} / \mathrm{L}$.

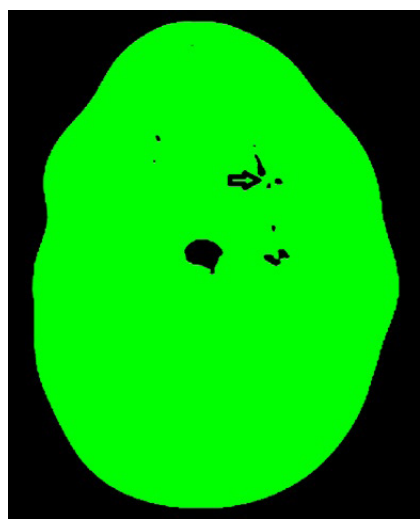

Figure 1: Axial CT of the neck demonstrating bilateral multiple collections in the neck. 
After immediate diagnosis the patient was posted for surgical decompression and tracheotomy to secure the airway. Separate stab incisions were made in relation to the submandibular space bilaterally and submental space. All decayed teeth were removed. With incising the connective tissue spaces pus was drained. The abscess incisions were left open and not packed, drains were placed (Figure 2). Due to the mediastinal spreading, intravenous administration of amoxicillin/ clavulanic acid 1200mg 4 times per day and Metronidazole 500mg 3 times per day were initiated. On the first postoperative day, a follow-up CT of the neck and thorax showed an increase of the mediastinal swelling. Laboratory work-up revealed high level of CRP 448 $\mathrm{mg} / \mathrm{L}$. The following two days showed a persistent increase in the mediastinal swelling and pleural effusion and a relative reduction in the parapharyngeal and retropharyngeal collections. On the third postoperative day, the patient was reoperated to relieve residual collections in the parapharyngeal region and around the hyoid bone. Irrigation with saline through the drains was done on a daily basis. The following days, the parapharyngeal, prevertebral and mediastinal collections increased and CT showed a radiologic picture consistent with mediastinitis (Figure 3 and Figure 4). Streptococcus anginosus was detected in a culture of the pus.

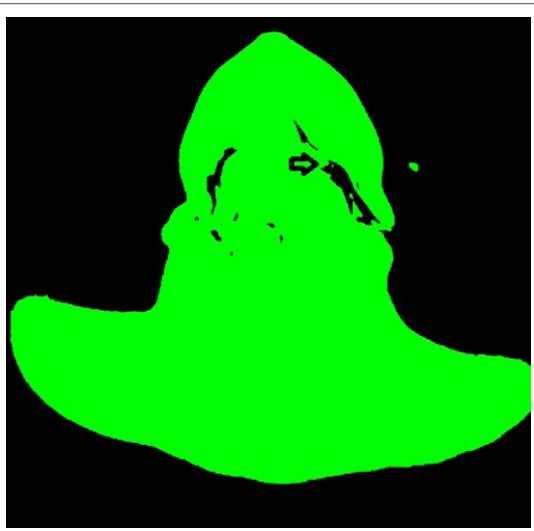

Figure 2: Correct drain locations verified by CT.

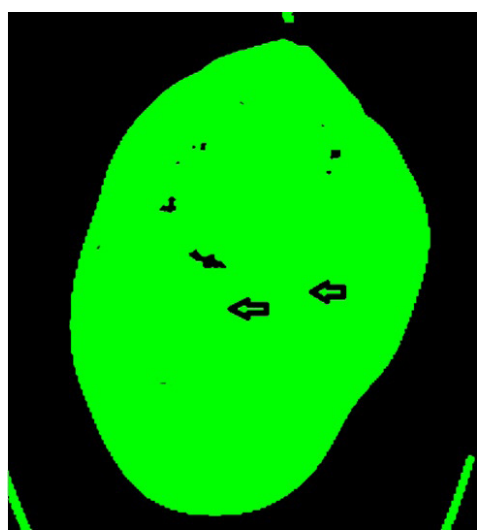

Figure 3: Increased parapharyngeal and prevertebral collections on a follow-up CT.

A week after the first intervention the patient was readmitted to the operating room. In cooperation with the cardiothoracic team an exploration of the mediastinal spaces with placement of three mediastinal drains was performed. Correct drain locations were verified by CT. Only then the infection parameters improved with a leucocyte count of $24.3109 / \mathrm{L}$ and CRP of $124 \mathrm{mg} / \mathrm{dl}$. On follow-up CT's adequate drainage of most of the collections was observed. On the $10^{\text {th }}$

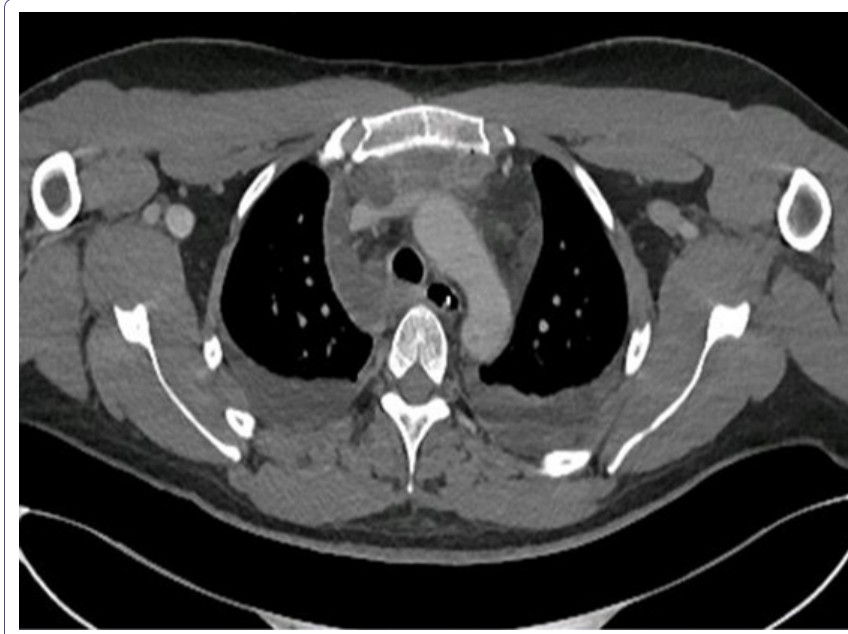

Figure 4: Axial CT of the chest showing mediastinal collections.

postoperative day the leucocyte count showed a drop to 19.9 109/L. The CRP raised again to $319 \mathrm{mg} / \mathrm{dl}$ with no evidence of a new focus. The following days the leucocytes count and CRP decreased and the patient was clinically improving. Four days later leucocyte count increased again and CT scan showed a persisting paravertebral collection with a persisting image of mediastinitis and pericardial effusion. Candida albicans was isolated in a pharyngeal swab and Fluconazol therapy was started. During the following week the patient significantly improved clinically and radiologically. Ten days after placement the thoracic drains were removed. After three weeks of intensive care, the patient was finally extubated and transferred to the ward with normal infection parameters. Four weeks after hospitalization the patient was dismissed.

\section{Case 2}

A 72-year-old obese male patient was referred from the Otorhinolaryngology (ENT) Department of the MUMC to the Department of Cranio-Maxillofacial Surgery complaining of a massive submandibular and submental swelling originating from the left lower jaw and neck which progressed to involve the contralateral side. The patient suffered from a morbid obesity $(>150 \mathrm{~kg})$, a status after myocardial infarction in 2015 and placement of a biventricular pacemaker thereafter. Furthermore a severe renal dysfunction (glomerular filtration rate 26), a cataract and an obstructive sleep apnea syndrome were documented. On examination, the patient was in poor general condition and slightly somnolent. He had respiratory distress and was toxic in appearance. His vital signs were: temperature $38.0^{\circ} \mathrm{C}$; pulse rate 65 beats/minute, blood pressure $110 / 60 \mathrm{mmHg}$ and a respiratory rate of 30 breaths/minute. Intraoral examination revealed decayed upper right first molar and lower right second molar teeth consistent with a history of not visiting a dentist in the last 6 years. Extraorally a diffuse reddness and swelling of the neck were visible; the swelling was tender on palpation. A CT of the neck showed extensive bilateral submandibular fluid collections (Figure 5). The blood values showed a high leucocyte count of 24.4 109/L and an elevated CRP of $336 \mathrm{mg} / \mathrm{dl}$.

An immediate diagnosis was made and the patient was transferred immediately for surgical decompression under general anesthesia. Multiple stab incisions were made in the submandibular region bilaterally and submentally to access the retro and parapharyngeal spaces. Pus was drained. Surgical extraction of decayed teeth was done simultaneously. The wounds were left open and drains were placed 
Citation: Kosba A, van Mierlo A, Dik E, Böckmann R, Kessler P (2017) Severe Life-Threatening Odontogenic Infections: Two Case Reports. J Infect Non Infect Dis 3: 021 .

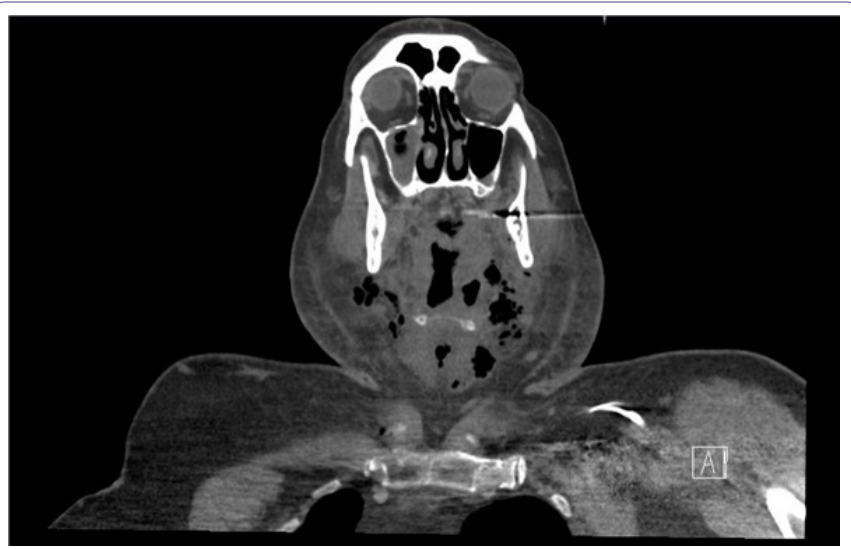

Figure 5: Multiple collections in the neck.

(Figure 6). The abcess cavities were irrigated with saline. After abscess drainage the patient was referred to the intensive care unit. Due to massive obesity the patient was kept intubated and ventilated with an oral tube. Intravenous administration of amoxicillin/clavulanic acid $1200 \mathrm{mg}$ twice daily coupled with metronidazole $500 \mathrm{mg}$ twice per day were given. Postoperative CT of the neck and thorax revealed a decrease in the submandibular swelling bilaterally, the left parapharyngeal and retropharyngeal collections. Streptococcus epidermidis and Streptococcus anginosus were isolated from the culture of pus. Infection parameters showed relative improvements on a daily based follow-up which was consistent with the follow-up CT scans on the $6^{\text {th }}$ and $9^{\text {th }}$ postoperative days. On the $11^{\text {th }}$ postoperative day, neck drains were removed. No evidence of collections was seen on the last CT scan and the patient was dismissed one week later.

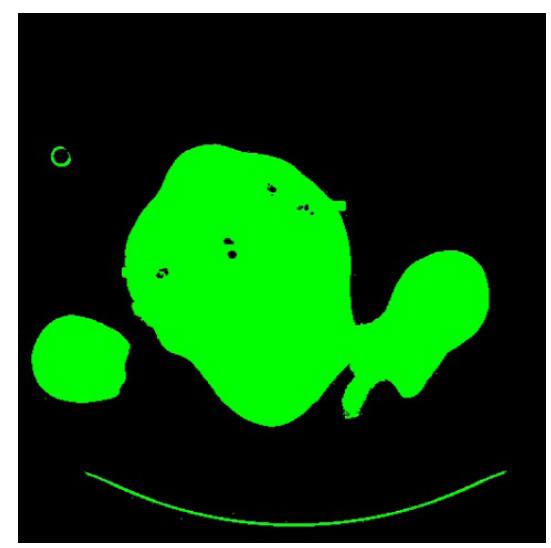

Figure 6: Postoperative verification of drain locations.

\section{Discussion}

Odontogenic infections are usually locally confined, self-limiting processes. However, under certain circumstances, they may break through the anatomical barriers and spread into distant spaces, resulting in severe fulminating infections. The clinical features, laboratory work-up and radiological assessment provide the necessary information to evaluate the severity and the extent of the infection. Despite the widespread utilization of antibiotics and improvements in healthcare services, odontogenic maxillofacial infections remain a common cause of morbidity and mortality worldwide [2]. At least in healthy individuals these infections can be mostly avoided by proper dental care and control. The therapy of extensive head and neck abscesses imposes substantial financial costs and may result in severe, even life-threatening complications, such as necrotizing fasciitis, airway obstruction, thrombosis of the cavernous sinus, orbital abscess, loss of vision, descending mediastinitis, cardiac tamponade and severe sepsis [8-12]. The frequency of odontogenic infections progression to Necrotizing Soft Tissue Infections (NSTIs) is approximately $1 \%$, however most cases of NSTIs are odontogenic in origin [13]. Early diagnosis and intervention is imperative in NSTIs, however, it is difficult to identify any specific predictive factors.

Flynn showed that fever, swelling, dysphagia and trismus were the symptoms most commonly observed in patients hospitalized for odontogenic infections [14]. It has also been demonstrated that strongly elevated white blood cell count and CRP are key findings in the decision to admit patients with odontogenic infection [14-16]. The combined presence of these symptoms and inflammatory markers is the classical criterion indicating the need for admission to ensure clinical treatment. Dental panoramic radiography is still regarded as the standard $\mathrm{x}$-ray to identify the cause of infection by providing good visualization of maxillary and mandibular dental structures, but provides little information about the severity or spread of the infection [17]. Contrast enhanced head and neck CT scan is indicated when the presence of an abscess in the fascial spaces of the head and neck, involving the parapharyngeal, submandibular and sublingual or masticator spaces, is suspected. CT also allows visualization of deep expansion of these spaces during extensive infection due to subcutaneous emphysema or abscess. Odontogenic infections occasionally spread to the deep fascial layers of the head and mainly the neck, evolving into severe life-threatening situations. These infections are more prevalent in developing countries [18]. Rana et al., have shown that $80 \%$ of neck space infection patients belong to lower socioeconomic class [19]. Both cases presented here reflect a problem of society. Despite health education and excellent medical services in developed countries, some individuals neglect their health and expose themselves to life-endangering ondontogenic infections. A maximum of interdisciplinary medical supply was necessary to treat these patients properly and save their lives.

Important contributions to processes of infectious spread in oral and maxillofacial surgery are poor oral hygiene, insufficient self-medication, non-compliant utilization of antibiotics and most important insufficient treatment of the underlying cause, mostly decayed teeth. Delayed referral to expert treatment adds up to the problem.

\section{Conclusion}

Early recognition of potential cases, appropriate referral, aggressive antibiotic and timely surgical intervention are very much important to reduce morbidity and mortality of such cases. As a safe and effective management protocol in cases of maxillofacial odontogenic infection, we propose early surgical management with incision and drainage and if indicated removal of the causative tooth or teeth as early as possible. The use of intravenous antibiotics with aerobic and anaerobic coverage and the adaptation of these antibiotics on the basis of sensitivity testing are essential pharmaceutical tools supporting the surgical treatment to prevent septical complications. Preventive dental care remains the best option available to mitigate the consequences of poor oral hygiene. Poor compliance among patient population for regular dental visits and oral hygiene maintenance emphasises the need for more sensitisation and education programs. 


\section{References}

1. Shah A, Ramola V, Nautiyal V (2016) Aerobic microbiology and culture sensitivity of head and neck space infection of odontogenic origin. Natl J Maxillofac Surg 7: 56-61.

2. Sato FR, Hajala FA, Freire Filho FW, Moreira RW, de Moraes M (2009) Eight-year retrospective study of odontogenic origin infections in a postgraduation program on oral and maxillofacial surgery. J Oral Maxillofac Surg 67: 1092-1097.

3. Brush JM, Treister NS (2010) Oral infections. In: Brush JM, Treister NS (eds.). Clinical Oral Medicine and Pathology. Humana Press, New York, USA Pg no: 83-88.

4. [No authors listed] (1992) Parameters of care for oral and maxillofacial surgery. A guide for practice, monitoring and evaluation (AAOMS Parameters of Care-92). American Association of Oral and Maxillofacial Surgeons. J Ora Maxillofac Surg 50: 1-174.

5. Singer AJ, Talan DA (2014) Management of skin abscesses in the era of methicillin-resistant Staphylococcus aureus. N Engl J Med 370: 1039-1047.

6. Fahimi J, Singh A, Frazee BW (2015) The role of adjunctive antibiotics in the treatment of skin and soft tissue abscesses: a systematic review and meta-analysis. CJEM 17: 420-432.

7. Topazian RG, Goldberg MH (1993) Oral and Maxillofacial Infections (3rdedn). Philadelphia, Saunders, USA. Pg No: 52: 528-529.

8. Chueng K, Clinkard DJ, Enepekides D, Peerbaye Y, Lin VYW (2012) An Unusual Presentation of Ludwig's Angina Complicated by Cervical Necrotizing Fasciitis: A Case Report and Review of the Literature. Case Reports in Otolaryngology 2012: 931350.

9. Jain S, Nagpure PS, Singh R, Garg D (2008) Minor trauma triggering cervicofacial necrotizing fasciitis from odontogenic abscess. J Emerg Trauma Shock 1: 114-118.
10. Yuka Sumi (2015) Descending necrotizing mediastinitis: 5 years of published data in Japan. Acute Medicine \& Surgery 2: 1-12.

11. Zachariades N, Vairaktaris E, Mezitis M, Rallis G, Kokkinis C, et al. (2005) Orbital abscess: Visual loss following extraction of a tooth--Case report. Oral Surg Oral Med Oral Pathol Oral Radiol Endod 100: 70-73.

12. Rallis G, Papadakis D, Koumoura F, Gakidis I, Mihos P (2006) Rare complications of a dental abscess. Gen Dent 54: 44-45.

13. Dillon JK, Zempleyni K (2015) How Often Do Odontogenic Infections Progress to Necrotizing Fasciitis and Can Progression be Predicted? J Oral and Maxillofac Surg 73: 39-40.

14. Flynn TR, Shanti RM, Levi MH, Adamo AK, Kraut RA, et al. (2006) Severe odontogenic infections, part 1: prospective report. J Oral Maxillofac Surg 64:1093-1103.

15. Ylijoki S, Suuronen R, Jousimies-Somer H, Meurman JH, Lindqvist C (2001) Differences between patients with or without the need for intensive care due to severe odontogenic infections. J Oral Maxillofac Surg 59: 867-873.

16. Storoe W, Haug RH, Lillich TT (2001) The changing face of odontogenic infections. J Oral Maxillofac Surg 59: 739-749.

17. Bondemark L, Jeppsson M, Lindh-Ingildsen L, Rangne K (2006) Incidental findings of pathology and abnormality in pretreatment orthodontic panoramic radiographs. Angle Orthod 76: 98-102.

18. Laskar HA, Karim HMR, Jamil M, Yunus M, Shunyu NB (2015) Diversified and Unusual Presentations of Neck Space Infections: Still a Big Concern for Physicians. J Maxillofac Oral Surg 14: 935-942.

19. Rana K, Rathor PK, Wadhwa V, Kumar S (2013) Deep neck infections: continuing burden in developing world. Int J Phonosurg Laryngol 3: 6-9. 